\title{
Bilateral arytenoid dislocation and fixation - a rare cause of difficult intubation
}

Hae Wone Chang, II OK Lee, Chunghoon Lee, Beong-gun Lim

Korea University Guro Hospital, Seoul, South Korea

\section{Background}

We presented of an experience of fiberoptic intubation for a patient with unrecognized bilateral arytenoid dislocation and fixation.

Case Report:

A 55-year old woman was scheduled for laparoscopic staging operation for endometrial cancer. Preoperative airway exam determined that Mallampati score was 2, and there were no risk factors related with the difficulty of airway. Her body weight, height, and BMI were $61 \mathrm{~kg}, 150 \mathrm{~cm}$, and $27.1 \mathrm{kgm}^{-2}$ respectively. Her American Society of Anesthesiology (ASA) physical status was 2. She had no symptoms related with laryngeal injury such as hoarseness, dyspnea, odynophagia and stridor. Her chest $X$ ray showed focal bronchiectasis and infiltration suggesting previous tuberculosis lesion in right lower lobe. The anesthesia was induced with propofol $2 \mathrm{mg} / \mathrm{kg}$ and rocuronium $0.6 \mathrm{mg} / \mathrm{kg}$, intravenously, and inhalation of $3 \%$ sevoflurane was used for maintenance. After obtaining complete neuromuscular blocking, intubation was attempted with direct laryngoscopy (Macintosh 3 laryngoscope blade). During endotracheal intubation, Cormack-Lehan laryngoscopy grade was 2 , and narrowed glottic opening was seen and both vocal cords were relatively fixed at paramedian position. Both 7.0 and $6.5 \mathrm{~mm}$ endotracheal tube could not be inserted. Under videolaryngoscopy (McGrath, Aircraft Medical, Edinburgh, UK) view, $6.0 \mathrm{~mm}$ tube was inserted into the trachea while counterclockwise 90 degree rotating the tip of endotracheal tube, but it was not further advanced enough, and it was positioned at $18 \mathrm{~cm}$ marking of level of patient's lip. We fixed the endotracheal tube at this point and decided to cancel the operation. We needed to evaluation for possibility of undiagnosed subglottic lesion. If we try repeated attempts of laryngoscopy, narrowed glottis and traumatized and edematous subglottic lesion may compromise the airway.

Learning points: Bilateral arytenoid dislocation and fixation is rare disease but challenging for intubation. It may impinge the tracheal tube advancing during general anesthesia or awake intubation. Selection of appropriate size of endotracheal tube, fiberscope size, intubation route and cooperation of patient are important factors for successful intubation.

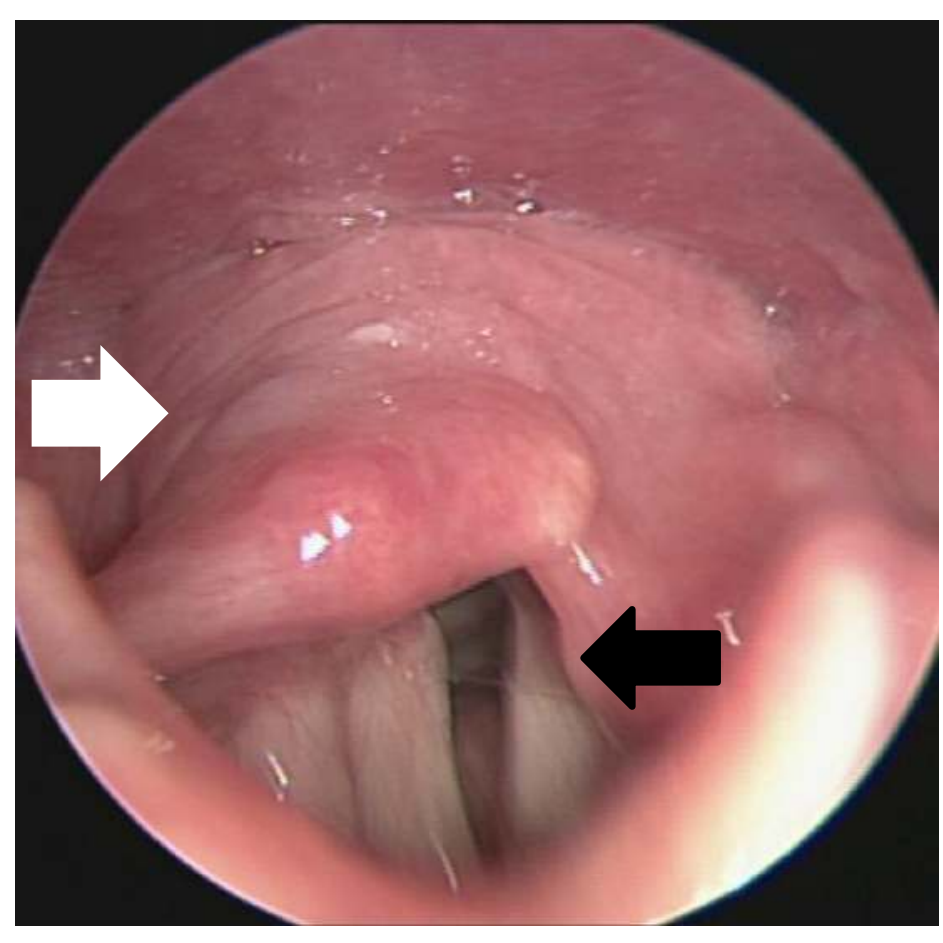

Fig 1. Endoscopic transnasal flexible laryngoscopic view. There is asymmetric anteromedial location of the right arytenoid cartilage (white arrow) relative to left arytenoid (black arrow).

Consultation was requested to outpatient clinic otolaryngologist. Her endoscopic transnasal flexible laryngoscopic finding suggested that decreased movement of vocal cord and asymmetric and dysmorphic arytenoid cartilages, and there is no supra or subglottic lesion (Fig 1). Neck computed tomography scan revealed that deformity of both arytenoid and cricoid cartilage with dysmorphic supraglottic area. There was no stenosis in her subglottic and trachea lesion (Fig 2). 3D CT scan showed no supra and subglottic lesion and dysmorphic arytenoid cartilages (Fig 3). Her EMG finding showed no neurologic deficit, ruled out recurrent laryngeal nerve injury. Conclusively, she was diagnosed with both arytenoid dislocation and fixation. After a few days, awake fiberoptic intubation was planned. A 4.2-mm fiberoptic bronchoscope was inserted through the left nostril and it was advanced into the nasopharynx and further down till the epiglottis and vocal cords were visualized. Similar to laryngoscopic finding, arytenoid cartilages were dysmorphic and fixed, and normal free movement of arytenoids was not shown. Two vocal cords were only partially abducted. We encouraged the patient to make a high pitched phonation. Fiberoptic bronchoscopy was gently passed through vocal cord, and 6.0-mm endotracheal tube was inserted over the bronchoscopy successfully. 6 hours of operation was uneventful. Discussion: Arytenoid abnormality is rarely associated with difficult intubation in previous reports, however, bilateral fixed morphology of the structures may hinder inserting appropriate size of endotracheal tube under general anesthesia. Careful history taking and physical examination as well as preoperative laryngoscopy and imaging study are helpful for planning of anesthesia of patients.

References: 1 . New insights into the pathomechanism of postintubation arytenoid subluxation. Anesthesiology. 1999 ;91:65966. Paulsen FP, Rudert HH, Tillmann BN.

a.

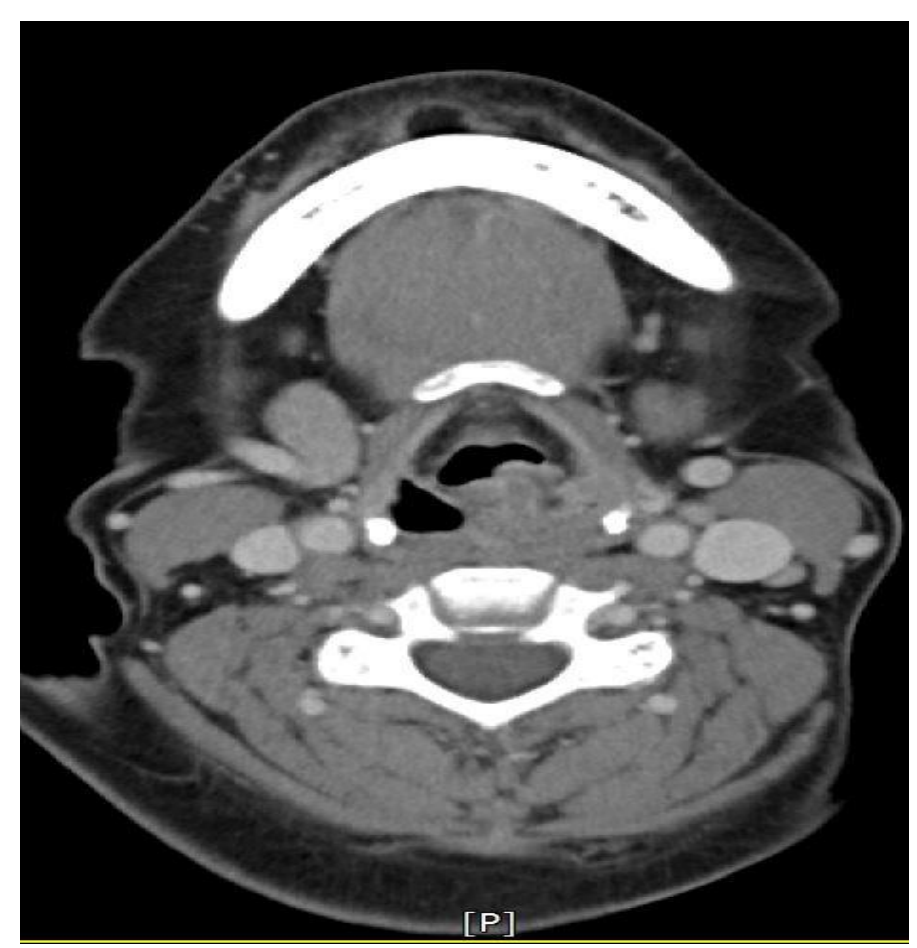

b.

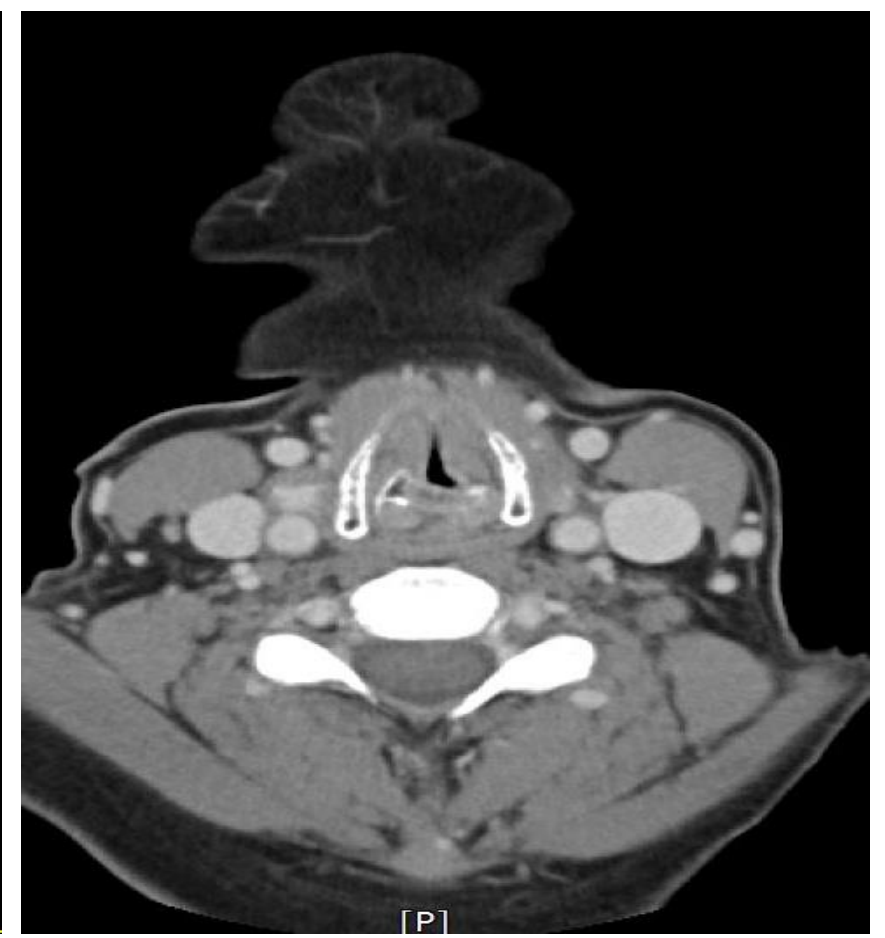

Fig 2 (a) supraglottic, and (b) vocal cord level in axial image, showed severely dysmorphic supraglottic lesion, and (C) right cricoarytenoid joint is superiorly positioned compared to left side in saggital image. c.
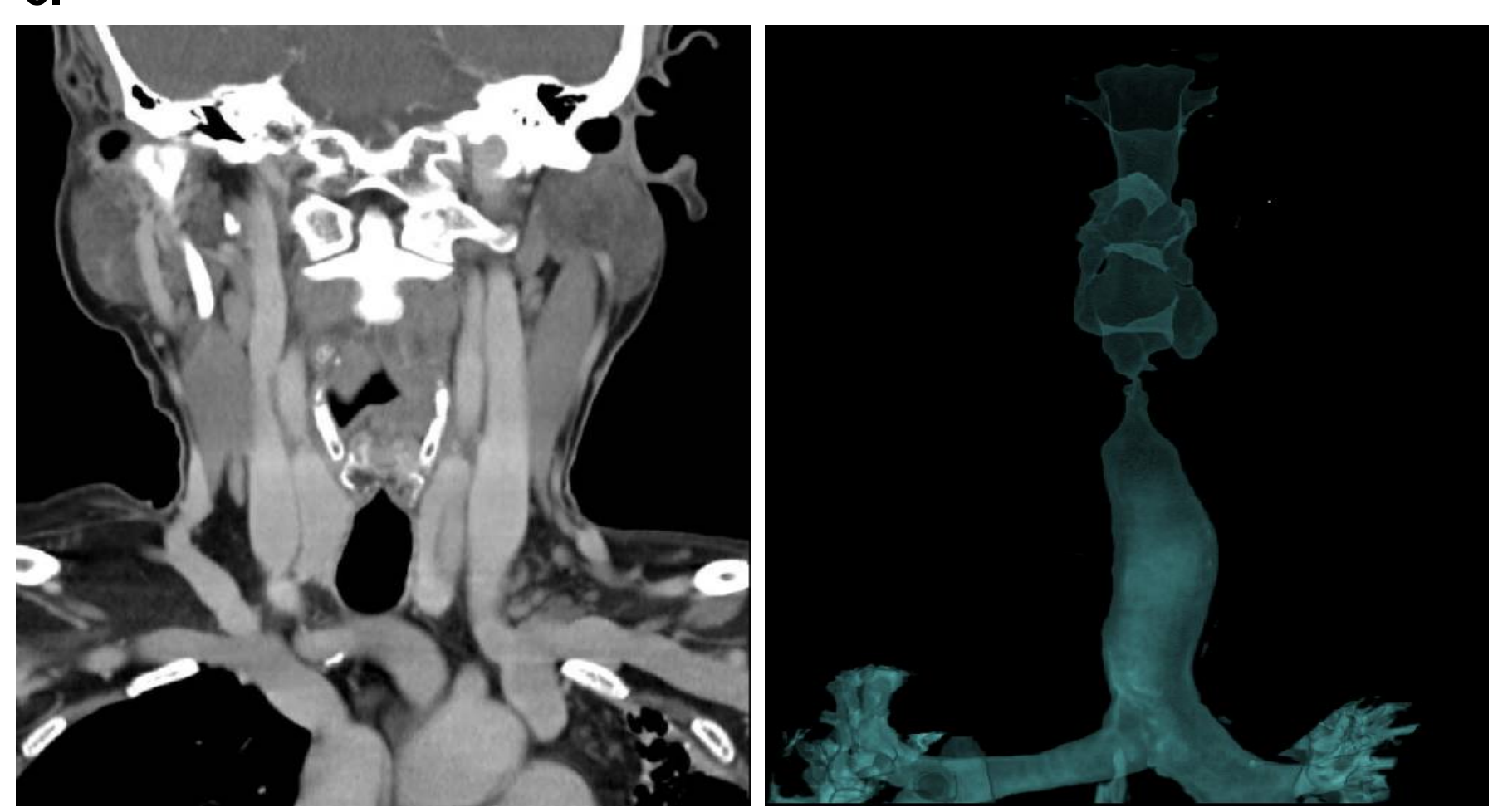

Fig 3. 3D CT image 